Präv Gesundheitsf 2022 · 17:44-50 https://doi.org/10.1007/s11553-021-00844-x Eingegangen: 30. Oktober 2020

Angenommen: 7. März 2021

Online publiziert: 6 . April 2021

(c) Der/die Autor(en) 2021

Julian Wangler · Michael Jansky

Zentrum für Allgemeinmedizin und Geriatrie, UNIVERSITÄTSMEDIZIN Mainz, Mainz, Deutschland

\title{
Potenziale der Bewegungs- förderung älterer Menschen im hausärztlichen Setting - eine explorative Interviewstudie mit Allgemeinmedizinern
}

oder Schwimmen. Ergänzend sollten ein maßvolles Krafttraining und unterstützende Aktivitäten für Beweglichkeit und Balance erfolgen $[4,13]$.

Aufgrund ihrer oft langjährigen $\mathrm{Pa}$ tientenkenntnis werden Hausärztinnen und Hausärzte als gut geeignete Bewegungs- und Gesundheitsberater auch und gerade für Personen im höheren Lebensalter angesehen [1, 3, 10, 11]. Hausärzte haben nicht nur die Möglichkeit, Empfehlungen und Verweise auszusprechen, sondern können konkrete physische Maßnahmen verschreiben, darunter Funktionstraining und seit 2012 ein sog. ,Rezept für Bewegung', das auch der Patientensensibilisierung dienen soll [12].

Bislang liegen für den deutschsprachigen Raum nur wenige Arbeiten vor, die sich mit der Frage beschäftigen, welche Rolle Hausärztinnen und Hausärzte bei der Bewegungsberatung und -verschreibung spielen [6, 10, 17]. Älteren Arbeiten zufolge sind niedergelassene Ärzte unterschiedlicher Facharztgruppen zwar davon überzeugt, dass viele Zivilisationskrankheiten durch Bewegung entscheidend positiv beeinflusst werden können, allerdings wurden z.T. Unsicherheiten im Hinblick auf die gesundheitsorientierte Bewegungsberatung artikuliert [2]. In Bezug auf die Wirksamkeit einer geleisteten Bewegungsberatung nahm in qualitativen Studien weniger als die Hälfte der Ärzte an, dass Patienten aufgrund dessen eine sportliche Aktivität aufnehmen würden $[3,18]$. Indes zeigten Patientenbefra- gungen, dass Personen, die eine solche Beratung von Haus- bzw. Fachärzten erhalten haben, sich gut angeleitet fühlen, um eine Lebensstiländerung $\mathrm{zu}$ initiieren [7]. Während einzelne Daten belegen, dass die Verschreibungspraxis bei Hausärzten hinsichtlich physischer Maßnahmen - etwa in Bezug auf die Ausstellung eines Rezepts für Bewegung - bislang vergleichsweise zurückhaltend ist, fehlt es an Erkenntnissen über Voraussetzungen und Barrieren der Bewegungsberatung [7, 18, 20].

Die vorliegende Arbeit soll einen Beitrag leisten, den Status quo des Themas Bewegungsförderung für ältere Menschen im hausärztlichen Setting zu explorieren.

\section{Methodik}

Die Studie basiert auf qualitativen, halbstandardisierten Leitfadeninterviews. Forschungsleitend waren die Fragestellungen, welche Potenziale Hausärztinnen und Hausärzte sehen, zur Bewegungsförderung im höheren Lebensalter beizutragen, welche Vorgehensweisen sie für Erfolg versprechend halten und welche Herausforderungen sie im Themenzusammenhang erleben. Vor dem Hintergrund der Erkenntnisse sollen Ansätze für eine Stärkung der hausärztlichen Versorgung abgeleitet werden. 


\begin{tabular}{ll} 
Tab. $\mathbf{1}$ & Soziodemografie der Befragungsstichprobe $(n=38)$ \\
\hline Alter & $\boldsymbol{\emptyset} \mathbf{5 3}$ Jahre \\
\hline Geschlecht & 19 männlich, 19 weiblich \\
\hline Niederlassungsform & 21 Einzelpraxis, 17 Gemeinschaftspraxis \\
\hline Praxisumgebung & 17 Landgemeinde/Kleinstadt, 13 Mittelstadt, 8 Großstadt \\
Status & 28 Praxisinhaber, 10 angestellte Ärzte \\
Weiterbildungshintergrund & 9 themennahe Fortbildungen, 5 sportmedizinische Weiterbildung, \\
& 3 geriatrische Weiterbildung
\end{tabular}

\section{Leitfaden und Rekrutierung}

Entlang der Forschungsfragen wurde ein kompakter, 24 Kernfragen umfassender Leitfaden erstellt (s. Anhang). Das Instrument wurde unter Berücksichtigung einer Literaturrecherche (hierv. a. die Expertise von Kleinert et al. [10]) und einer Vorstudie mit älteren Patienten [20] sowie im Zuge erster Gespräche abgeleitet.

Bei der Rekrutierung wurden bestimmte soziodemografische Merkmale bewusst variiert (vgl. - Tab. 1), um ein heterogenes Sample zu erhalten. Insgesamt wurden 53 Hausärztinnen und Hausärzte in Rheinland-Pfalz und Hessen kontaktiert, wobei 38 Interviews geführt wurden (19 pro Bundesland).

\section{Durchführung und Sample}

Im Vorfeld erhielten die Interviewten eine Aufklärung über das Gesprächsthema sowie eine schriftliche Einverständniserklärung. Die Interviews fanden zwischen März und September 2020 statt und wurden in 17 von 38 Fällen telefonisch durchgeführt (40 bis $70 \mathrm{~min}$ ). Die theoretische Sättigung wurde erreicht. @ Tab. 1 zeigt das gewonnene Sample.

\section{Auswertung}

Die im Anschluss an die Datenerhebung erstellten Transkripte wurden im Team mithilfe einer qualitativen Inhaltsanalyse nach Mayring [14] ausgewertet (Software MAXQDA). Zunächst wurden die bedeutungstragenden Grundaussagen herausgearbeitet, anschließend weiter abstrahiert und zusammengefasst und schließlich ein Kategoriensystem erstellt, das sich eng am Leitfaden orientierte und mit Fortgang der Auswertung wiederholt geprüft und ggf. modifiziert wurde. Im Mittelpunkt stand, die unterschiedlichen
Sichtweisen und Erfahrungen logisch zu kategorisieren.

\section{Einhaltung ethischer Richtlinien}

Im Zuge der vorzustellenden Studie wurden keine Patientendaten erhoben oder klinische Tests durchgeführt. Es handelt sich um durchgehend pseudonymisierte Interviews mit Hausärztinnen und Hausärzten, bei denen grundlegende Einstellungen und Erfahrungen in Bezug auf die Bewegungsförderung ermittelt wurden. Dennoch wurde eine Rückversicherung bei der Ethikkommission des Bundeslandes Rheinland-Pfalz eingeholt, die ergab, dass ein Ethikvotum aufgrund des Studiendesigns nicht erforderlich ist.

\section{Ergebnisse}

\section{Bedeutung von Bewegungsförde- rung im höheren Lebensalter}

Nahezu sämtliche Interviewte $(n=36)$ sehen die Bewegungs- und Gesundheitsförderung als (sehr) wichtiges Aufgabenfeld der (Primär-)Versorgung. Dies gilt insbesondere für die Gruppe älterer Patienten, wo der Wert von Fitness und Sport nicht nur in reiner Gesundheitsvorsorge bestehe, sondern für „das subjektive Wohlbefinden ..., für die Stärkung von Alltagsfähigkeiten und das Gefühl, sich noch Dinge zutrauen zu können ... nicht hoch genug eingeschätzt werden“ könne (I-11w).

Trotz der Betonung des Themas Bewegung weist mehr als die Hälfte der Interviewten $(n=21)$ darauf hin, dass es im Praxisalltag oft nicht machbar sei, eine „vollwertige, längerfristige Beratung zu leisten" (I-19m). Bei älteren Menschen gestalte sich dies besonders herausfordernd, da mehr Spezifika (z.B. Vorerkrankungen, Passgenauigkeit zu beste- henden Therapien) berücksichtigt werden müssten und es einen größeren $\mathrm{Be}$ treuungsbedarf gebe. Rund ein Drittel der Ärzte $(n=14)$ gibt an, ältere Personen häufiger im Hinblick auf körperliche Betätigung zu beraten, bei einem weiteren Drittel $(n=13)$ ist dies gelegentlich der Fall.

Grundsätzlich gefragt, sehen die Interviewten verschiedene Möglichkeiten, zur Bewegungsförderung beizutragen:

Das Wichtigste ist in meinen Augen zu sensibilisieren, anzuraten, zu empfehlen, vor allem bei vorerkrankten Patienten. (I14m)

Man kann natürlich selbst als Coach einsteigen, indem ein Programm mit konkreten Zielen vereinbart wird. Das setzt aber Zeitreserven und eine Verlaufskontrolle voraus. $(\mathrm{I}-19 \mathrm{~m})$

Ich glaube, was den Hausarzt auszeichnet, ist das Lotsen, das Vermitteln zu anderen Ärzten, Therapeuten, Anbietern. Der Hausarzt als Sprungbrett für Patienten. $(\mathrm{I}-17 w)$

\section{Anlass und Fokus der Bewegungsberatung}

Aus ihrer Erfahrung berichten die Interviewten, dass die Ausgangspunkte für eine Bewegungsberatung bei älteren Patienten stark unterschiedlich sein können.

Manchmal sind es Leute, die merken, dass ihre Fitness leidet, und sie wollen etwas dagegen unternehmen. Manchmal sind es chronisch Kranke. Es gibt auch diejenigen, die auf der Suche nach einer sinnvollen Beschäftigung sind. (I-32m)

Ein größerer Teil der Befragten $(n=25)$ schätzt, dass das Thema Bewegung und Sport in den meisten Fällen von ihrer Seite initial angesprochen wird und sich auf dieser Grundlage eine Beratung ergibt. Dies gilt insbesondere dann, wenn Bewegung als längerfristige Maßnahme eine Stabilisierung oder Verbesserung des Gesundheitszustandes verspricht (z. B. Übergewicht, Diabetes).

Viele Hausärzte (30) sind der Auffassung, dass die Bereitschaft älterer Menschen, sich regelmäßig zu bewegen, in „weiten Teilen vorhanden" sei (I-15w). Allerdings bestehe bei diesen oft „Un- 
Präv Gesundheitsf 2022 · 17:44-50 https://doi.org/10.1007/s11553-021-00844-X

(c) Der/die Autor(en) 2021

\section{J. Wangler $\cdot$ M. Jansky}

\section{Potenziale der Bewegungsförderung älterer Menschen im hausärztlichen Setting - eine explorative Interviewstudie mit Allgemeinmedizinern}

\section{Zusammenfassung}

Hintergrund. Gerade im höheren Lebensalter kann regelmäßige körperliche Aktivität einen wertvollen Beitrag zur Gesundheitsvorsorge und zum Erhalt eines selbständigen Lebens leisten. Das hausärztliche Setting gilt als gut geeignet, um ältere Patienten kompetent zu beraten, sie auf geeignete Bewegungsformate hinzuweisen und zu einer längerfristigen Wahrnehmung selbiger zu motivieren.

Ziel der Arbeit. Die Studie soll einen Beitrag leisten, den Status quo des Themas Bewegungsförderung für ältere Menschen im hausärztlichen Setting zu explorieren.

Material und Methoden. Zwischen März und September 2020 wurden 38 qualitative Einzelinterviews mit Hausärzt*innen in Rheinland-Pfalz und Hessen geführt.
Ergebnisse. Die Interviewten zeigen ein ausgeprägtes $\mathrm{Ma}$ an Bewusstsein und Sensitivität in Bezug auf die Gesundheitsund Bewegungsförderung im höheren Lebensalter. Viele Ärzte sind engagiert, wenn es darum geht, geeignete Aktivitäten für ältere Patienten zu identifizieren und diese nachhaltig zu einer Teilnahme zu bewegen. Einige Ärzte arbeiten eng mit Gesundheitsund Sportakteuren vor Ort zusammen. Die Interviewten benennen Herausforderungen, insbesondere ein Fehlen adäquater Strukturen zur Bewegungsförderung. Einem Teil der Hausärzte mangelt es an einem Überblick, welche Bewegungsangebote in der Umgebung angeboten werden; Kooperationen mit Gesundheitsanbietern sind nicht immer gegeben.
Schlussfolgerung. Hausärzt*innen sollten in einer aktiven Rolle im Bereich der Bewegungsund Gesundheitsförderung bestärkt werden. Damit sie effektiv auf Angebote verweisen bzw. Patienten dorthin vermitteln können, kommt es darauf an, das hausärztliche Setting in ein kommunales Netzwerk de Prävention und Gesundheitsförderung einzubinden. Gezielte Schulungen können das hausärztliche Team darin unterstützen, Praxisbesuche älterer Patienten systematisch zu nutzen, um auf den Wert körperlicher Aktivität hinzuweisen.

Schlüsselwörter Bewegung · Gesundheitsförderung . Prävention · Sport · Kursangebote

\section{Potentials of physical activity promotion for older adults in family practice-an exploratory interview study with general practitioners}

\section{Abstract}

Background. Physical activity can support the maintenance of an independent and healthy life, especially in older individuals. The results show that family physicians are in a particularly favorable position to advise older patients regarding physical exercise, fitness and health services to the elderly and motivate them to take part in these over the longer term.

Objectives. The study contributes to exploring the status quo of the topic of physical activity promotion for older people in family practice. Materials and methods. Between March and September 2020, 38 qualitative individual interviews were conducted with family physicians in Rhineland-Palatinate and Hesse (Germany).
Results. The interviewees show a pronounced level of awareness and sensitivity with regard to the topic of health and physical activity promotion for older people. Many physicians are committed to identifying suitable activities for older patients and motivating them to participate in the long term. Some physicians work closely with local health and sports stakeholders. The interviewees also name challenges, in particular a lack of adequate structures to promote physical activity. Some of the family physicians lack an overview of the types of sports, fitness or health offers that are locally available; cooperation with health providers is not always given.

Conclusion. Family physicians should be encouraged to actively promote physical activity. In order to enable them to effectively refer older patients to sports, fitness or health offers, it is important to integrate the general practitioner setting in local and municipal networks for health promotion. Targeted training courses can support the whole practice staff to systematically use office visits by older patients to point out the value of physical activity.

Keywords

Exercise $\cdot$ Health promotion $\cdot$ Preventive medicine. Sports $\cdot$ Courses sicherheit, inwiefern sich dies negativ auf bestimmte Leiden auswirken" (I27w) oder Sportangebote vorhandene Beschwerden verstärken könnten. Insofern sei ein wichtiges Bedürfnis dieser Patientenklientel, „eine Art von Sicherheitsgefühl zu bekommen“" (I-34w). Aus der Erfahrung wird der eigene Rat von den meisten Patienten ernst genommen und unterbreitete Empfehlungen angenommen.
Das Gros der Hausärzte berichtet, die älteren Patienten vorgeschlagenen Bewegungsarten seien in erster Linie Aktivitäten wie Schwimmen, Radfahren, Walken, (moderates) Joggen oder seniorenfreundliche Gymnastik- und Fitnesskurse.

Wichtig sind schonende und zugleich ganzheitliche Sportarten. (I-34w)
Die Verbindung von Ausdauer, Kraft und Beweglichkeit machen den idealen Sport für Senioren aus. (I-5m)

Die meisten Interviewten $(n=26)$ beschränken sich darauf, allgemeine Ratschläge oder Empfehlungen zu geben; ein kleinerer Teil $(n=11)$ stellt in Einzelfällen Bewegungspläne auf und definiert gemeinsam mit dem Patienten zu erreichende Ziele. Dabei werden konkrete, 
z. T. erstattungsfähige Maßnahmen verordnet, also etwa von Funktionstraining oder Reha-Sport Gebrauch gemacht. Verbreitet ist die Unterstützung eigener Empfehlungen durch Hilfsmittel wie Broschüren und Informationsblätter (Seniorensport, seltener kommunale Angebote) oder Verweise auf Gesundheitsseiten im Internet. Einige Interviewte $(n=4)$, darunter v. a. sportmedizinisch weitergebildete Ärzte, agieren in Gesundheitsnetzwerken und vermitteln Patienten bei Bedarf und Interesse gezielt an ausgewählte Gesundheits- und Sportzentren sowie Physiotherapeuten weiter.

\section{Vorgehen bei Bewegungsberatung}

Die Ärzte artikulieren, dass es wichtig sei, bei älteren Patienten nicht nur durch Aufklärung Einsicht in den Nutzen von Bewegung zu schaffen, sondern mit passgenauen Empfehlungen das angesprochene „Sicherheitsbedürfnis“ (I-34w) zu adressieren, mit der Wahrnehmung körperlicher Aktivitäten keine Risiken einzugehen.

Dazu gehört auch, dass man sich der $\mathrm{Sa}$ che langsam nähert und hineintastet. (I$17 w)$

Ebenfalls sei zentral, nicht lediglich an die Aufnahme von Bewegung zu appellieren, als vielmehr eine ,intrinsische Motivation“ zu fördern, die schnell als „selbstverständlicher Tages- oder Wochenrhythmus verinnerlicht" werden kann (I-20m).

Richtig hast $d u$ es gemacht, wenn der Patient Bewegung nicht als etwas Aufoktroyiertes empfindet. (I-16w)

Sport soll Spaß machen. Natürlich versuche ich auch herüberzubringen, dass es eine soziale Aktivität sein kann. (I-38m)

Es wird dafür plädiert, dass der Hausarzt bei Nahelegung bestimmter Aktivitäten antizipiert, „wie passgenau das Angebot zur Persönlichkeit des Patienten“ (I-20m) ist und wie gut sich dieses ,in den Alltag und in die Lebenslage“" (I-36w) integrieren lässt.

Zentral sei es, „ein klares Bewusstsein dafür zu haben“, inwiefern eine zu empfehlende oder zu verordnende Bewegungsaktivität rein physische Effekte zeitigen soll oder ob es auch um psychosoziale Stärkung geht (I-5m).

Ältere Patienten sind manchmal von depressiven Phasen betroffen. Denen kann man gut durch Bewegung entgegenwirken.... Bei Manchen steht die reine Frage der Fitness gar nicht im Vordergrund. (I28m)

Viele Ärzte betonen auch die Wichtigkeit einer realistischen Umsetzbarkeit der Empfehlungen. Daher müssten Bewegungsangebote „möglichst niedrigschwellig“ wahrnehmbar sein (I-32m).

Wo Bewegungsberatung stattgefunden hat, beschreibt der Großteil des Samples positive Erfahrungen und Effekte. Beobachtet wurde, dass bei einer Annahme der Bewegungsaktivitäten ältere Patienten „vielleicht sogar rascher und längerfristiger als Jüngere" eine Routine entwickelten und sich dabei wohl fühlten (I-34w).

\section{Herausforderungen und Optimierungsansätze}

Als problematisch wird indes empfunden, dass die Bewegungsberatung „längst nicht überall da stattfinden kann, wo sie nötig wäre“ (I-19m).

Das Hauptproblem sind definitiv nicht negative Effekte oder mangelnde Bereitschaft der Patienten, sondern dass wir eher selten dazu kommen, uns diesem Feld gebührend $z u$ widmen. $(I-17 w)$

Dies wird einerseits mit Zeitknappheit begründet, andererseits mit dem Fehlen adäquater Strukturen und Anlaufstellen für Bewegungsförderung. Dies geht einher mit einer aus Sicht vieler Hausärzte nicht zufriedenstellenden Honorierungssituation.

Ich stehe damit alleine da. Wo sind die helfenden Hände? Wo sind systematische Begleitprogramme, an die ich den Patienten überführen kann? (I-22m)

Abseits von irgendwelchen Sportinitiativen, die es vielleicht hier und da auf kommunaler Ebene gibt, steht da kein flächendeckendes Supportnetz zur Verfügung. (I-27w)

Mehrere Hausärzte kritisieren, dass sie sich bei Fragen der Gesundheitsförde- rung „ein wenig im Stich gelassen“ fühlten (I-1m) und alleine keinen ausreichenden Überblick über lokale Sportangebote hätten, an die sie Patienten schnell und unkompliziert verweisen könnten.

Es fehlt an Transparenz und an Ansprechpartnern, gerade wenn es um Fit-Sein im Alter geht.... Ich sehe da die Kommunen in der Pflicht, mehr zu tun. (I-22m)

Einem Teil der Interviewten sind Verschreibungsmöglichkeiten wie die Ausstellung des Rezepts für Bewegung $(n=21)$ oder durch die Krankenkassen bezuschusste SPORT PRO GESUNDHEITKursangebote $(n=18)$ bekannt. Beim Verordnen von Funktionstraining ist eine Reihe von Ärzten $(n=16)$ nach eigener Aussage „vergleichsweise verhalten“ (I$14 \mathrm{~m})$. Als Begründung hierfür räumt ein Teil der Befragten ein, sich bei der Bewegungsberatung und -verschreibung „nicht vollständig kundig“ $\mathrm{zu}$ fühlen (I-35m) oder Verschreibungsoptionen „nicht immer gleich in Erwägung zu ziehen" (I-23w). Auch sehen sich einige Ärzte nicht primär für die Verschreibung physischer Maßnahmen zuständig und verweisen lieber an Fachärzte oder Physiotherapeuten.

Mit Blick auf mögliche Optimierungsansätze artikulieren die Befragten neben dem Wunsch nach geeigneten Strukturen und Programmen ein „breiteres Angebot an attraktiven Weiterbildungsformaten für Ärzte und Praxispersonal“ (I29w). Gerade mit Blick auf letzteres werden Delegationsmöglichkeiten ins Auge gefasst.

Weitergebildete Praxismitglieder können Patienten auch unterstützen, informieren, motivieren, dafür braucht es nicht zwangsläufig den Hausarzt. (I-29w)

Einige Ärzte sehen Chancen in Gesundheits-Apps und der von der Bundesregierung kürzlich geschaffenen Möglichkeit, lizensierte digitale Gesundheitsanwendungen auf Rezept verordnen zu können.

Zum Beispiel habe ich gehört, dass da eine vielversprechende Adipositas-App kommen soll. Das könnte einiges für die Situation von Hausärzten erleichtern. (I$5 m$ ) 
Auch wird angeregt, dass Krankenkassen ihre älteren Versicherten stärker auf Präventionsangebote hinweisen. Dies wäre ein Beitrag, um Ärzte beim Suchen und Finden von passgenauen Angeboten zu entlasten.

\section{Diskussion}

\section{Zusammenfassung und Befunde anderer Studien}

Im Zuge der Interviewstudie wurden das Bewusstsein und die Einstellung eines gemischten Samples von Hausärztinnen und Hausärzten gegenüber der Bewegungsförderung für ältere Menschen sichtbar. Bestehende Netzwerke, Verhaltensmuster sowie Förderfaktoren und Herausforderungen wurden durch die Interviews synthetisiert. Diese zeigen bei Hausärztinnen und Hausärzten insgesamt ein ausgeprägtes $\mathrm{Maß}$ an Kenntnis und Sensitivität in Bezug die Gesundheits- und Bewegungsförderung im höheren Lebensalter. Viele der Befragten sind engagiert, wenn es darum geht, geeignete Aktivitäten für ältere Patienten zu identifizieren und diese zu einer nachhaltigen Teilnahme zu motivieren. Einige Ärzte arbeiten mit Sport- und Gesundheitsakteuren aus der Umgebung zusammen, um an konkrete Angebote zu verweisen und deren Passgenauigkeit für Patienten sicherzustellen.

Die Interviewten benennen auch Hürden und Herausforderungen. So sieht ein Teil der Ärzte im Fehlen adäquater Strukturen und Programme zur Bewegungsförderung ein erhebliches Defizit; analog dazu wird das Vergütungssystem kritisiert. Hinzu kommt, dass es einer Reihe von Hausärzten an einem Überblick mangelt, welche Fitness- und Sportangebote in der Umgebung angeboten werden; Kooperationen mit Gesundheitsanbietern sind nicht immer gegeben. Ferner macht ein Teil der Befragten nur begrenzt von Verordnungsmöglichkeiten wie Funktionstraining Gebrauch und ist mit dem Rezept für Bewegung nicht vertraut.

Viele der Resultate fügen sich in die bestehende Studienlage, der zufolge sich für die hausärztliche Versorgung große Potenziale im Bereich von Prävention und
Gesundheitsförderung zeigen, aber auch Problematiken wie Zeit- und Ressourcenknappheit $[18,19]$ sowie Mangel an finanzieller Vergütung [21]. Auch besteht die Schwierigkeit, dass spezielle Beratungs- und Versorgungslösungen nicht breit bekannt oder nur wenig genutzt sind [7, 9]. Zudem sind lokale Kurs- und Unterstützungsangebote aber auch Beratungsmaterialien schwer überschaubar bzw. zugänglich [19]. Frühere Studien haben ermittelt, dass aufärztlicher Seite teils Unsicherheiten im Hinblick auf gesundheitsorientierte Bewegungsberatung bestehen [2]. Nichtsdestotrotz eruierte eine Umfrage unter Patienten, die eine solche erhalten hatten, dass die ärztliche Unterstützung als (sehr) hilfreich beurteilt wird [7].

In einer Befragung älterer Patienten zeigte sich die Problematik, dass diese zwar häufig Interesse an Bewegungsangeboten haben, es ihnen jedoch nicht selten an einem kompetenten, glaubwürdigen Mediator fehlt, der diese Angebote bedarfsorientiert vermitteln kann [20]. Aus Sicht der Befragten genießen Hausärztinnen und Hausärzte als Vermittler im Bereich Gesundheitsförderung hohes Vertrauen. Allerdings gaben nur etwa $40 \%$ an, dass ihnen der Hausarzt schon bestimmte Gesundheitsangebote empfohlen hat. Wurden diese Empfehlungen aber ausgesprochen, haben die meisten Befragten das entsprechende Angebot nicht nur ausprobiert, sondern längerfristig wahrgenommen.

Eine Reihe von Arbeiten regen an, das hausärztliche Setting konsequenter zu nutzen, um zu einer körperlichen Aktivierung älterer Personen beizutragen $[6,15]$. Exemplarisch herausgegriffen sei die Studie von Prüfer et al., die großes Potenzial darin sehen, die hausärztliche Präventionsarbeit in ein kommunales System der Gesundheitsförderung einzubetten [17]. Flankierend können gezielte Schulungen dazu beitragen, eine effektive Bewegungsförderung in hausärztlichen Praxen zu verankern [15].

\section{Stärken und Schwächen}

Obwohl ein heterogenes Hausärztesample rekrutiert werden konnte, weist die Studie verschiedene Limitationen auf.
Neben der begrenzten Fallzahl und dem den Einzelfall betrachtenden qualitativen Ansatz handelt es sich um einen regionalen Rekrutierungsschwerpunkt.

Hinsichtlich der Mitwirkungsbereitschaft der Gesprächspartner sollte bedacht werden, dass verstärkt Hausärzte teilgenommen haben könnten, bei denen ein thematisches Interesse vorliegt. Ferner wurde ein großer Teil der Interviews telefonisch geführt, was gegenüber Faceto-face-Befragungen Einschränkungen für die Aussagekraft der Ergebnisse mit sich gebracht haben kann.

Die Befragung war ausschließlich auf Bewegungsförderung konzentriert. In der Versorgungsrealität ist diese Dimension oft mit anderen Gebieten verknüpft, z. B. Lebensstil und Ernährung. Solche Zusammenhänge wurden hier nicht beleuchtet.

\section{Schlussfolgerungen}

Das hausärztliche Setting ist gut geeignet, um ältere Patienten in Bezug auf gesundheitsförderliche Bewegung $\mathrm{zu}$ beraten und $\mathrm{zu}$ einer längerfristigen Wahrnehmung von individuell geeigneten Bewegungsangeboten zu motivieren [19]. Diese Vermittlerrolle sollte durch einen Mehrklang von Maßnahmen gestärkt werden:

- Hausärztinnen und Hausärzte sollten auf lokaler/kommunaler Ebene stärker in formelle wie informelle Netzwerke zur Stärkung der Gesundheitsförderung eingebunden werden, sodass sie u. a. einen Überblick über vorhandene Angebote (z. B. in Vereinen) haben und unkompliziert darauf verweisen können [17].

- Bei der Bereitstellung bedarfsgerechter Gesundheitsangebote für ältere Menschen ist eine Kooperation zwischen Hausärzten und anderen Gesundheitsakteuren (Physiotherapeuten, Trainer) von Vorteil. Prüfer et al. haben eine Veränderung struktureller Rahmenbedingungen angeregt, konkret einen Abbau von Kooperationsbarrieren und eine genaue Definition der Rollen der beteiligten Akteure sowie ausreichende Ressourcen [17]. 
- Hausärztinnen und Hausärzte sollten bestärkt werden, von bereits vorhandenen Möglichkeiten, Funktionstraining oder Reha-Sport zu verschreiben, Gebrauch zu machen. Anlässe wie Vorsorgeuntersuchungen lassen sich systematisch nutzen, um auf den Wert kontinuierlicher körperlicher Aktivität hinzuweisen und Empfehlungen auszusprechen $[6,10]$.

- Themenspezifische Weiterbildungsformate zur Bewegungsförderung bei Älteren sollten dem hausärztlichen Team verstärkt zugänglich gemacht werden [15], um Kompetenzen zu fördern und Delegationspotenziale für das Praxispersonal aufzuzeigen.

- Nicht zuletzt sollte erwogen werden, die Vergütungsbedingungen von hausärztlichen Beratungsleistungen zur Gesundheitsförderung durch Schaffung aufwandsadäquater Abrechnungsziffern anzupassen.

\section{Fazit für die Praxis}

- Das hausärztliche Setting ist gut geeignet, um ältere Patienten in Bezug auf gesundheitsförderliche Bewegung zu beraten und zu einer längerfristigen Wahrnehmung von individuell geeigneten Bewegungsangeboten zu motivieren. Hausärztinnen und Hausärzte sollten daher in einer aktiven Rolle im Bereich der Bewegungs- und Gesundheitsförderung bestärkt werden.

- Damit Hausärzte effektiv auf Bewegungsangebote in der Umgebung verweisen bzw. Patienten dorthin vermitteln können, kommt es darauf an, das hausärztliche Setting in ein kommunales Netzwerk der Prävention und Gesundheitsförderung einzubinden.

- Gezielte Schulungen können das hausärztliche Team darin unterstützen, Praxisbesuche älterer Patienten systematisch zu nutzen, um auf den Wert körperlicher Aktivität hinzuweisen, Informationen zu vermitteln und konkrete Empfehlungen auszusprechen.

\section{Korrespondenzadresse}

Dr. phil. Julian Wangler

Zentrum für Allgemeinmedizin und Geriatrie, UNIVERSITÄTSMEDIZIN Mainz

Am Pulverturm 13, 55131 Mainz, Deutschland julian.wangler@unimedizin-mainz.de

Funding. Open Access funding enabled and organized by Projekt DEAL.

\section{Einhaltung ethischer Richtlinien}

Interessenkonflikt. J. Wangler und M. Jansky geben an, dass kein Interessenkonflikt besteht.

Bei der vorliegenden Interviewstudie wurden keinerlei sensible Patientendaten erhoben oder klinische Tests durchgeführt. Eine Rückversicherung bei der Ethikkommission des Bundeslandes Rheinland-Pfalzergab, dass ein Ethikvotum nicht erforderlich ist.

\section{Anhang}

\section{Interviewleitfaden}

Bedeutung von Bewegungsförderung im höheren Lebensalter

- Welche Bedeutung messen Sie dem Thema Bewegung und Sport in der Patientenversorgung bei?

- Welche Rolle spielt der Hausarzt, wenn es um das Thema Gesundheitsund Bewegungsförderung geht? Inwieweit sehen Sie den Hausarzt als primären oder wichtigen Ansprechpartner für Bewegung und Sport? Inwiefern ist es wünschenswert, sich als Hausarzt mit diesem Thema zu befassen und Patienten zu beraten?

- Wie ist das bei Ihnen im Praxisalltag: Welchen Stellenwert hat da das Thema Gesundheits- und Bewegungsförderung?

- Welche Möglichkeiten und Potenziale sehen Sie als Hausarzt, um zur Bewegungsförderung bei älteren Menschen beizutragen? (Um diese Patientengruppe soll es im Folgenden gehen.)

- Wie häufig beraten Sie ältere Patienten mit Blick auf Bewegung bzw. Sport?
Anlass, Umstände und Fokus der Bewegungsberatung

- Wie ergibt sich diese Beratung üblicherweise? Kommen die Patienten auf Sie zu und bitten um Informationen/Empfehlungen oder sprechen Sie das Thema Bewegung bzw. Sport von sich aus an?

- Was ist der genuine Anlass (Prävention, vorhandene Erkrankungen bzw. therapeutische Maßnahmen, Rehabilitation)?

- Wie nehmen Sie Bedarfe und Bedürfnisse nach Bewegungsförderung bei älteren Patienten i.d. R. wahr? Welche Wichtigkeit hat hier der hausärztliche Rat?

- Welche Art von Bewegung legen Sie älteren Menschen nahe?

- Betreiben Sie ausschließlich allgemeine Bewegungsberatung und geben Empfehlungen oder stellen Sie zusammen mit dem Patienten auch konkrete Bewegungspläne/ gemeinsame Ziele auf?

- Inwiefern verordnen Sie konkrete physische Maßnahmen oder vermitteln Patienten an bestimmte Gesundheitsakteure? Weshalb (nicht)?

- Inwiefern nutzen Sie bei der Bewegungsberatung Hilfsmittel wie Broschüren, Informationsblätter oder geben Empfehlungen für bestimmte Internetseiten?

\section{Vorgehen bei Bewegungsbe-} ratung, Erfahrungen mit und beobachtete Effekte

- Was sind Spezifika von älteren Patienten, wenn es um Bewegung und Sport geht? Worauf sollte man als Hausarzt bei der Bewegungsberatung oder bei konkreten Empfehlungen oder Verordnungen achten?

- Welche Erfahrungen haben Sie grundsätzlich mit der Bewegungsberatung älterer Menschen gemacht? Welche Effekte haben Sie beobachtet?

- Wie oft trägt die von Ihnen geleistete Bewegungsberatung Ihren Beobachtungen nach Früchte?

- Wie gut lassen sich ältere Patienten Ihrer Erfahrung nach zu mehr Sport und Bewegung motivieren? Was läuft 
gut, was weniger gut? Wie ist die langfristige Teilnahmebereitschaft?

- Haben Sie bestimmte Vorgehensweisen oder Strategien, um möglichst zum Erfolg einer Bewegungsberatung beizutragen?

\section{Überblick über lokale Bewegungs- angebote und Kooperation mit Gesundheitsakteuren}

- Welche Angebote zur Bewegungsund Gesundheitsförderung für ältere Patienten gibt es bei Ihnen in der Umgebung? Inwiefern würden Sie sagen, dass Sie hier einen guten Überblick haben? Auf welchem Weg erfahren Sie üblicherweise von Angeboten wie Sport-, Fitness- oder Bewegungskursen?

- Inwiefern arbeiten Sie beim Thema Bewegungsförderung (für ältere $\mathrm{Pa}$ tienten) mit anderen Gesundheitsakteuren zusammen (z. B. Sportvereine, Gesundheitszentren, Fitnessstudios, kommunale Bewegungsinitiativen, Physiotherapeuten)? Weshalb (nicht)? Inwieweit wäre eine (stärkere) Kooperation in diesem Bereich sinnvoll?

- Wie beurteilen Sie die Zusammenarbeit mit Fachärzten, wenn es um das Thema Bewegungsförderung geht?

\section{Erlebte Herausforderungen und Optimierungsansätze}

- Welche Herausforderungen und Probleme nehmen Sie wahr, wenn es um das Thema Bewegungsförderung älterer Menschen geht?

- Was sollte oder müsste Ihrer Ansicht nach getan werden, damit Sie bzw. Hausärzte insgesamt ältere Patienten noch besser im Bereich der Gesundheits- und Bewegungsförderung unterstützen können?

- Es gibt den Vorschlag, dass Hausärzte möglichst systematisch mit Anbietern von Gesundheits- und Bewegungskursen zusammenarbeiten sollten (z. B. Fitnessstudios, Krankenkassen, Volkshochschule), um Patienten ggf. schneller auf geeignete Angebote hinweisen oder dorthin vermitteln $\mathrm{zu}$ können. Wie stehen Sie dazu?

- Sollten Hausärzte in Zukunft eine stärkere Mediatorenrolle einnehmen, wenn es um die Vermittlung von (älteren) Patienten zu Gesundheitsund Bewegungsangeboten geht? Unter welchen Voraussetzungen wäre dies für Sie denkbar?

Open Access. Dieser Artikel wird unter der Creative Commons Namensnennung 4.0 International Lizenz veröffentlicht, welche die Nutzung, Vervielfältigung, Bearbeitung, Verbreitung und Wiedergabe in jeglichem Medium und Format erlaubt, sofern Sie den/die ursprünglichen Autor(en) und die Quelle ordnungsgemäß nennen, einen Link zur Creative Commons Lizenz beifügen und angeben, ob Änderungen vorgenommen wurden.

Die in diesem Artikel enthaltenen Bilder und sonstiges Drittmaterial unterliegen ebenfalls der genannten Creative Commons Lizenz, sofern sich aus der Abbildungslegende nichts anderes ergibt. Sofern das betreffende Material nicht unter der genannten Creative Commons Lizenz steht und die betreffende Handlung nicht nach gesetzlichen Vorschriften erlaubt ist, ist für die oben aufgeführten Weiterverwendungen des Materials die Einwilligung des jeweiligen Rechteinhabers einzuholen.

Weitere Details zur Lizenz entnehmen Sie bitte der Lizenzinformation auf http://creativecommons.org/ licenses/by/4.0/deed.de.

\section{Literatur}

1. Becker S, Huy C, Brinkhoff KP et al (2007) Sport, Bewegung und Gesundheit im mittleren und höheren Erwachsenenalter. Gesundheitswesen 69(7):401-407

2. Braumann KM, Reer $R$, Schuhmacher E (2001) Die Einschätzung der Bedeutung von Sport und Bewegung als Mittel der Therapie bei niedergelassenen Ärztinnen und Ärzten in Hamburg. Dtsch ZSportmed 52(5):175-179

3. Bücker B, Mai A, Klaassen-Mielke R et al (2017) Einstellungen von Hausärzten zu einem Bewegungsprogramm für mobilitätseingeschränkte Ältere (HOMEfit).ZAllg Med 93(11):466-472

4. Bundesministerium für Gesundheit (2018) Nationale Empfehlungen für Bewegung und Bewegungsförderung. https://www. bundesgesundheitsministerium.de/service/ begriffe-von-a-z/b/bewegungsempfehlungen html.Zugegriffen: 30. Okt. 2020

5. Dapp U, Lorentz C, Laub S et al (2009) Im Alter aktiv und gesund leben - Ergebnisse einer repräsentativen Seniorenbefragung. Z Gerontol Geriatr 42(3):245-255

6. Gabrys L, Jordan S, Behrens Ketal (2016) Prävalenz, zeitliche Trends und regionale Unterschiede ärztlicher Bewegungsberatung in Deutschland. Dtsch ZSportmed 67(3):53-58

7. Gubisch R, Hoppe I, Vogt Let al (2014) Wirksamkeit des Rezepts für Bewegung aus Patientensicht: Ärztliche Beratung und Handlungskonsequenzen. Dtsch ZSportmed 56(7-8):206

8. Heath G, Parra DC, Sarmiento OL (2012) Evidencebased intervention in physical activity: lessons from around the world. Lancet 380:272-281

9. Intorp SK (2015) Pilotstudie zur körperlichen Aktivität. Universität Heidelberg, Heidelberg
10. Kleinert J, Zepp C, Glöckler K et al (2015) Aus der Arztpraxis in den Sportverein? Herausforderungen an eine ärztliche Präventionsempfehlung zur Veränderung des Bewegungsverhaltens. DSHS, Köln

11. Krüger-Band H (2011) Prävention: Der Hausarzt als Gesundheits-Coach. Dtsch Arztebl 108(41):16

12. Löllgen H, Wismach J, Kunstmann W (2013) Das Rezept für Bewegung - Einsatzmöglichkeiten für Arzt und Patient. Klinikarzt 42(9):416-420

13. Malik SH, Blake H, Suggs LS (2014) A systematic review of workplace health promotion interventions for increasing physical activity. Br J Health Psychol 19(1):149-180

14. Mayring P (2010) Qualitative Inhaltsanalyse. Grundlagen und Techniken, 11. Aufl. Beltz, Weinheim

15. Peters S, Schwab M, Faller H et al (2017) Schulung für Ärzte zur Bewegungsförderung bei Älteren. Präv Gesundheitsf 12(3):218-226

16. Pfeifer K, Rütten A (2017) Nationale Empfehlungen für Bewegung und Bewegungsförderung: Konzepte, Methoden, Ergebnisse. Gesundheitswesen 79(S01):S2-S3

17. PrüferF, JoosS, Milksch A (2015) Die Rolle des Hausarztes in der kommunalen Gesundheitsförderung. Präv Gesundheitsf 10(2):180-185

18. Ruhmann L (2014) Befragung von ausgewählten Berliner Ärztinnen und Ärzten für eine Evaluation des Rezeptes für Bewegung in Berlin. Charité Universitätsmedizin, Berlin

19. Tulloch H, Fortier M, Hogg W (2006) Physical activity counseling in primary care: Who has and who should be counseling? Patient Educ Couns 64(1-3):6-20

20. Wangler J, Jansky M (2020) Die Bedeutung des hausärztlichen Settings für die Bewegungs- und Gesundheitsförderung im höheren Lebensalter - Ergebnisse einer Befragung. Präv Gesundheitsf 15(2):188-193

21. Yarnall K, Pollak KI, Østbye T et al (2003) Primary care: is there enough time for prevention? Am J Public Health 93(4):635-641 